\title{
REVIEWING THE REVIEW PROCESS: EXAMINING DECISION-MAKING IN PRACTICE
}

\author{
Carolyn Anderson and Anna van der Gaag \\ Department of Speech and Language Therapy \\ University of Strathclyde \\ Southbrae Drive \\ Glasgow G13 1PP \\ email: carolyn.anderson@strath.ac.uk
}

\begin{abstract}
A national multi-disciplinary audit showed variation in SLT practice on the decision to review pre-school children or place them on waiting lists following initial assessment. Audit evidence suggests some reasons for these differences and contributing factors are illustrated by case history examples. Current health-care policies encourage practitioners to make decisionmaking transparent to consumers but this process is problematic if there is a lack of evidence to support principled choice. An outline is presented to enable therapists to gather their own evidence for review outcomes in daily practice.
\end{abstract}

\section{Introduction}

Variation in practice has been observed across many health-care services in the UK (Scally and Donaldson 1998). Speech and language therapy is therefore no exception (van der Gaag et al. 1999c). Differences in resource levels, staff workload, management practice and population characteristics account for some of this variation, and to some extent at least, this variation may be unavoidable. However, if health-care professionals are to pursue the principles of quality improvement, then minimising avoidable variation across the country must be one objective, as it is one way of ensuring that users of services receive the same service regardless of where they live. This paper attempts to explore variation in one small but crucial aspect of SLT practice: the use of the review category.

The use of the review category appears to be one area of SLT practice where there is considerable variation. This observation is based on data generated by the Royal College of Speech and Language Therapists' (RCSLT) national audit of services to preschool children with speech and language difficulties. The methodology and results of this audit are reported in full elsewhere (van der Gaag et al. 1998, 1999a, 1999b, Anderson and van der Gaag 1999). The audit was carried out in six districts across the UK between 1997 and 1998 . Therapists were asked to record information on referral and selection procedures for new referrals over a six-month period. They recorded data on the selection of children for intervention and the factors which were taken into consideration when making decisions about entry into therapy. 


\section{Results}

Analysis of data from the six sites was based on information on 648 audit forms from speech and language therapists (see table 1). More than $50 \%$ of the children referred in these districts were over three years old. In the majority of cases, the referrals were considered appropriate in terms of both the timing of the referral and the reason for the referral. However only a third of children were considered a priority for intervention by the speech and language therapy service. Of the children who were not considered as priority cases, $82 \%$ were placed on review for an average of four months and $12 \%$ were discharged. Parental concern was often cited as a reason for monitoring a child's development by placing the child on review.

Table 1. Analysis of therapist audit forms

\begin{tabular}{|c|c|c|c|c|c|c|c|}
\hline Districts & 1 & 2 & 3 & 4 & 5 & 6 & Total \\
\hline Therapists' audit forms & 59 & 74 & 103 & 58 & 167 & 187 & 648 \\
\hline Age of referrals $(\%)$ : & $(\%)$ & & & & & & Mean \\
\hline under 2 years & 5 & 5 & 8 & 5 & 11 & 3 & 6 \\
\hline $2-4$ years & 76 & 83 & 73 & 69 & 84 & 82 & 80 \\
\hline $4-5$ years & 19 & 12 & 18 & 26 & 5 & 15 & 14 \\
\hline Referral information: & $(\%)$ & & & & & & \\
\hline referrals necessary & 81 & 96 & 96 & 88 & 97 & 91 & 93 \\
\hline priority for therapy & 37 & 20 & 35 & 38 & 31 & 40 & 34 \\
\hline $\begin{array}{l}\text { Outcome of referral for } \\
\text { non-priority children }(66 \%) \text { : }\end{array}$ & $(\%)$ & & & & & & \\
\hline difficulty severe/very severe & 0 & 2 & 1 & 0 & 3 & 1 & 1 \\
\hline difficulty moderate/mild & 70 & 83 & 84 & 81 & 88 & 74 & 82 \\
\hline difficulty age-appropriate & 30 & 15 & 14 & 19 & 9 & 25 & 17 \\
\hline Action for non-priority children: & $(\%)$ & & & & & & \\
\hline discharged & 19 & 17 & 12 & 6 & 4 & 17 & 12 \\
\hline placed on a waiting list* & 0 & 27 & 37 & 72 & 5 & 7 & 19 \\
\hline placed on review* & 81 & 80 & 75 & 56 & 96 & 83 & 82 \\
\hline $\begin{array}{l}\text { Outcome of referral for } \\
\text { priority children }(34 \%) \text { : }\end{array}$ & $(\%)$ & & & & & & \\
\hline difficulty severe/very severe & 32 & 33 & 47 & 41 & 27 & 33 & 34 \\
\hline difficulty moderate/mild & 68 & 67 & 53 & 59 & 73 & 67 & 66 \\
\hline Action for priority children: & $(\%)$ & & & & & & \\
\hline immediate intervention & 77 & 7 & 11 & 9 & 8 & 45 & 28 \\
\hline within $4-8$ weeks & 23 & 33 & 33 & 41 & 10 & 47 & 33 \\
\hline more than 8 weeks & 0 & 60 & 47 & 50 & 81 & 7 & 39 \\
\hline
\end{tabular}

* denotes categories are not mutually exclusive

Some of the variation across the six districts reflected differences in geography, management practice and local policy on referral procedures. The number of returns from each site also varied. One site had staffing difficulties during the audit period, which may have accounted for the small number of returns. The age at which children were referred to speech and language therapy was similar across the six districts. District 4 had the highest proportion of children over four years (26\%). Fewer than $11 \%$ of children referred were under two years in all locations. 
In all districts, under $20 \%$ of children referred were discharged during the six month period (with under $7 \%$ in Districts 4 and 5 ). In five districts, more than three quarters of all children referred were placed on review, and less than $40 \%$ were placed on the waiting list. Interestingly, in three districts, significant numbers of children appeared in both waiting list and review categories as therapists did not always treat these as mutually exclusive. For example, in District $4,56 \%$ of children were placed on review and $72 \%$ were placed on the waiting list.

Five districts considered $30-40 \%$ of children to be a priority for therapy (with $20 \%$ considered as such in District 2). Of those children who were not considered a priority, over $70 \%$ of this group were assessed as having a moderate or mild difficulty. In Districts 1 and 6 , over $25 \%$ of children were age-appropriate on assessment.

Patterns of intervention also showed considerable variation between locations. Immediate intervention accounted for $77 \%$ of children in District 1, 45\% in District 6 and around $10 \%$ in the remaining four districts. However, these districts showed a higher proportion of children who waited more than eight weeks for intervention.

\section{Case examples}

The audit results provide some evidence that therapists make the choice between review and waiting list placement for a variety of good reasons, e.g. the child's age, the severity of their condition and parental concerns. Two case histories are useful in illustrating the main types of decision-making which were typically reported from healthcare trusts.

The first case is Daniel who is seen for initial assessment at 3;09 years of age. His therapist notes that he has a moderate speech difficulty with some expressive language delay. As the department's policy is to offer immediate therapy only to children who are considered a high priority at assessment, the therapist arranges to review Daniel's progress in 4 months. As the therapist judges that Daniel will need direct therapy in future, his name is added to the waiting list in anticipation of the review outcome. This arrangement is agreed with Daniel's mother and she is advised about some general language stimulation activities with a handout to support this information. The review period will enable the therapist to judge how effective the indirect approach has been through parental intervention. This approach allows the department to plan management of caseloads and reduce crisis management.

The second case is William who is 3;03 years old. His speech is also assessed as moderately affected and he has additional expressive language delay. William's therapist decides that he is likely to need therapy and places his name on the waiting list for therapy. However, as this list is quite long and she is concerned to monitor his development, she also arranges to review his progress in four months. She explains the situation to his mother and gives her some advice on language activities. This therapist makes her decisions based on a range of criteria relating to the individual. For example, she would be more likely to review a child if they were under 2;06 years with ageappropriate verbal comprehension and poor speech but would offer therapy to a child with delayed receptive language at this age. Other candidates for review would include a child who has made spontaneous progress on the waiting list or where major changes are currently affecting the child's life such as starting nursery or the arrival of a new sibling. If the child is within the normal range for development but the parents are anxious, advice and review may also be used to monitor the situation. 


\section{Discussion}

This audit constituted a baseline audit, which examined local practices and produced useful feedback to the participating sites on the structure, processes and outcomes pertinent to the delivery of their services.

In terms of the implications of the audit, a number of trends have emerged. Firstly, the results highlight the variation in practice across the six sites. These variations are likely to reflect differences in management practice, prioritisation systems, size and location of site and level of experience of staff, e.g. Districts 1 and 4 differed in their use of waiting lists for non-priority children - District 1 placed children on review and District 4 placed them on a waiting list. It is important to note that these differences between districts were explicit and agreed before the commencement of the audit.

The results have raised questions about the nature of the review category as one aspect of a prioritisation system. One of the questions raised is whether therapists use review as a method of monitoring a child's progress when the child is unlikely to need therapy or whether it is routinely used as a method of checking on a child's development when the therapist expects that the child will need intervention. The variations revealed by the audit show how a process, which is designed to be used to allow monitoring in low priority cases, has become a method whereby caseloads can be controlled and case numbers manipulated. If there are good reasons why a child's progress should be monitored then this should be made explicit. But if review is used as a device to delay entry into a system when SLTs judge that intervention will be needed then clients may be disadvantaged in accessing the service. This type of management may also disguise the extent to which resources affect decision-making. If resources are limited, the decision on review must be more accurate in determining which children would benefit most from direct therapy and which would be adequately provided for by a review process.

Generally there is a lack of research evidence to enable therapists to make informed decisions on the choice between review and intervention. Research has shown that there is a reduction in the incidence of expressive language delay after the age of two years (Law et al. 2000), suggesting some spontaneous improvement takes place without intervention and it could be argued that this supports the decision to place some children on review. The STEP project (Glogowska et al. 2000) suggested that the majority of children with early speech and language delay in their sample continued to have difficulties a year after initial assessment regardless of whether they had received small amounts of therapy or 'watchful waiting' (review). This new evidence may prompt a radical rethink about the way in which decisions on review and intervention are made, with further clinical research needed to interpret factors such as the timing, duration, frequency and type of intervention.

Further questions are raised in regard to the review category in a prioritisation system. These include consideration of whether the extent of its use is determined by the level of experience of staff. It may be that newly qualified therapists need more explicit training in this area, with recognition that this may be part of a process of experiential development in decision-making. Similarly therapists understand that, in some cases, they need to review their hypotheses about a child's difficulties over time. In services where the review category is not used (e.g. see Stringer 1999), the extent of the responsibility afforded to parents becomes an issue. Therapists cannot assume that all parents can be actively involved in making decisions about whether or not their child needs further assessment and intervention. There may be logistic, cultural or economic reasons which mitigate against this. By discussing the decision-making process with the child's parents 
and others involved such as health visitors, general practitioners (GPs) and nursery staff, therapists are more likely to ensure that children do not slip out of the system by default.

Procedures for how to record the information over time would enable therapists to gather evidence for their own decision-making procedures. Information on the use of the review category could be recorded, using established audit procedures (van der Gaag et al. 1999b), e.g. therapists in a department could agree guidelines for review following consultation on criteria for this category. Guidelines would allow therapists to apply principled decision-making on whether therapy, review or discharge would be appropriate for each child over a chosen time period. This would allow evaluation of the effectiveness of the service and provide information to inform future decision-making for reviews. This audit showed disparity in provision or therapy access which may reflect resource allocation differences between districts. Evidence gathered by SLTs might also support the need for greater provision of service resources in some areas.

\section{Conclusion}

The audit shows variation in the use of the review category. The majority of preschool children referred to the audited SLT services were placed on review, following initial assessment. Factors such as severity of difficulty, age and parental concerns were cited by therapists in deciding between offering intervention and monitoring progress. Two examples were used to illustrate the reported qualitative factors which led to some of the variations between districts in the use of the review category. Other variations may have been due to factors related to the districts themselves, such as size and locally determined procedures. The variations observed in this audit suggest that therapists may use review as a method of checking a child's progress in low priority cases or as a 'holding' category for the majority of cases where therapy may be required. Recent research (Glogowska et al. 2000) supports what experienced SLTs know, i.e. most preschool children judged by therapists to have speech and language difficulties will require some type of intervention at some point.

Decisions on review should be made explicit within the service to ensure consistency of provision and support newly qualified therapists in practice (see also Pickstone 1997). This process would enable therapists to reflect on their current practice in this area. By monitoring review outcomes in daily practice, therapists could generate their own evidence of the use of this category in providing access to therapy. By examining decision-making in this process, SLTs can make their reasons transparent not only to themselves but to their clients. This examination should lead to clarification of entry into the service and reduce variations between districts due to reasons such as lack of funding for services.

\section{Acknowledgements}

The audit was funded by the Department of Health. The views expressed in this paper are entirely those of the authors. The authors would like to express their thanks to Pauline McCartan, Anne McDade and Dot Reid (who were members of the RCSLT Audit Team) and to the therapists, health visitors, GPs and parents who took part in the audit. 


\section{References}

Anderson, C. and van der Gaag, A., 1999, An examination of the pattern of preschool referrals to speech and language therapy. Child Language Teaching and Therapy, 16, 59-71.

Glogowska, M., Roulstone, S., Enderby, P. and Peters, T., 2000, Randomised controlled trial of community-based speech and language therapy in pre-school children. British Medical Journal, 321, 923-26.

Law, J., Boyle, J., Harris, F., Harkness, A. and Nye, C., 2000, Prevalence and natural history of primary speech and language delay: findings from a systematic review of the literature. International Journal of Language and Communication Disorders, 35, 165-188.

Pickstone, C., 1997, Weighting, not waiting. Human Communication, 6(2), 23-24.

Scally, G. and Donaldson, L., 1998, Clinical governance and the drive for quality improvement in the NHS British Medical Journal, 317,61-66.

Stringer, H., 1999, Are three-year-olds predictable? Bulletin of the Royal College of Speech and Language Therapists, 570, 12.

van der Gaag, A., McCartan, P., McDade, A., Reid, D., 1998, An audit tool for health visitors and speech and language therapists working with the pre-school population. International Journal of Language and Communication Disorders, 33, 37-41.

van der Gaag, A., Reid, D., McCartan, P. and McDade, A., 1999a, The Development of an Audit Tool for Speech and Language Therapists and Members of the Primary Healthcare Team (Report to the Department of Health).

van der Gaag, A., McCartan, P., McDade, A. and Reid, D., 1999b, The Early Communication Audit Manual (London: Royal College of Speech and Language Therapists).

van der Gaag, A., McLoone, P., Reid, D. 1999c, Speech and language therapy caseloads in seven districts in the UK. Journal of Management in Medicine, 13, 23-32. 